\title{
A GENERALIZATION OF A CONTRACTION PRINCIPLE IN PROBABILISTIC METRIC SPACES. PART II
}

\author{
DOREL MIHET
}

Received 7 June 2004 and in revised form 3 December 2004

A fixed point theorem concerning probabilistic contractions satisfying an implicit relation, which generalizes a well-known result of Hadžić, is proved.

\section{Preliminaries}

In this section we recall some useful facts from the probabilistic metric spaces theory. For more details concerning this problematic we refer the reader to the books $[1,3,9]$.

1.1. $t$-norms. A triangular norm (shortly $t$-norm) is a binary operation $T:[0,1] \times[0,1] \rightarrow$ $[0,1]:=I$ which is commutative, associative, monotone in each place, and has 1 as the unit element.

Basic examples are $T_{L}: I \times I \rightarrow I, T_{L}(a, b)=\operatorname{Max}(a+b-1,0)$ (Eukasiewicz $t$-norm), $T_{P}(a, b)=a b$, and $T_{M}(a, b)=\operatorname{Min}\{a, b\}$. We also mention the following families of $t$ norms:

(i) Sugeno-Weber family $\left(T_{\lambda}^{S W}\right)_{\lambda \in(-1, \infty)}$, defined by $T_{\lambda}^{S W}=\max (0,(x+y-1+\lambda x y) /$ $(1+\lambda))$

(ii) Domby family $\left(T_{\lambda}^{D}\right)_{\lambda \in(0, \infty)}$, defined by $T_{\lambda}^{D}=\left(1+\left(((1-x) / x)^{\lambda}+((1-y) /\right.\right.$ $\left.\left.y)^{\lambda}\right)^{1 / \lambda}\right)^{-1}$

(iii) Aczel-Alsina family $\left(T_{\lambda}^{A A}\right)_{\lambda \in(0, \infty)}$, defined by $T_{\lambda}^{A A}=e^{-\left(|\log x|^{\lambda}+|\log y|^{\lambda}\right)^{1 / \lambda}}$.

Definition $1.1[2,3]$. It is said that the $t$-norm $T$ is of Hadžic-type (H-type for short) and $T \in \mathscr{H}$ if the family $\left\{T^{n}\right\}_{n \in N}$ of its iterates defined, for each $x$ in $[0,1]$, by

$$
T^{0}(x)=1, \quad T^{n+1}(x)=T\left(T^{n}(x), x\right), \quad \forall n \geq 0,
$$

is equicontinuous at $x=1$, that is,

$$
\forall \mathcal{E} \in(0,1) \exists \delta \in(0,1) \quad \text { such that } x>1-\delta \Longrightarrow T^{n}(x)>1-\varepsilon, \quad \forall n \geq 1 .
$$

There is a nice characterization of continuous $t$-norms $T$ of the class $\mathscr{H}$ [8]. 
(i) If there exists a strictly increasing sequence $\left(b_{n}\right)_{n \in N}$ in $[0,1]$ such that $\lim _{n \rightarrow \infty} b_{n}=$ 1 and $T\left(b_{n}, b_{n}\right)=b_{n} \forall n \in N$, then $T$ is of Hadžić-type.

(ii) If $T$ is continuous and $T \in \mathscr{H}$, then there exists a sequence $\left(b_{n}\right)_{n \in N}$ as in (i).

The $t$-norm $T_{M}$ is an trivial example of a $t$-norm of $H$-type, but there are $t$-norms $T$ of Hadžić-type with $T \neq T_{M}$ (see, e.g., [3]).

Definition 1.2 [3]. If $T$ is a $t$-norm and $\left(x_{1}, x_{2}, \ldots, x_{n}\right) \in[0,1]^{n}(n \in N)$, then $T_{i=1}^{n} x_{i}$ is defined recurrently by 1 , if $n=0$ and $T_{i=1}^{n} x_{i}=T\left(T_{i=1}^{n-1} x_{i}, x_{n}\right)$ for all $n \geq 1$. If $\left(x_{i}\right)_{i \in N}$ is a sequence of numbers from $[0,1]$, then $T_{i=1}^{\infty} x_{i}$ is defined as $\lim _{n \rightarrow \infty} T_{i=1}^{n} x_{i}$ (this limit always exists) and $T_{i=n}^{\infty} x_{i}$ as $T_{i=1}^{\infty} x_{n+i}$. In fixed point theory in probabilistic metric spaces there are of particular interest the $t$-norms $T$ and sequences $\left(x_{n}\right) \subset[0,1]$ such that $\lim _{n \rightarrow \infty} x_{n}=1$ and $\lim _{n \rightarrow \infty} T_{i=1}^{\infty} x_{n+i}=1$. Some examples of $t$-norms with the above property are given in the following proposition.

Proposition 1.3 [3]. (i) For $T \geq T_{L}$ the following implication holds:

$$
\lim _{n \rightarrow \infty} T_{i=1}^{\infty} x_{n+i}=1 \Longleftrightarrow \sum_{n=1}^{\infty}\left(1-x_{n}\right)<\infty .
$$

(ii) (1.3) also holds for $T=T_{\lambda}^{S W}$.

(iii) If $T \in \mathcal{H}$, then for every sequence $\left(x_{n}\right)_{n \in N}$ in I such that $\lim _{n \rightarrow \infty} x_{n}=1$, one has $\lim _{n \rightarrow \infty} T_{i=1}^{\infty} x_{n+i}=1$.

(iv) If $T \in\left\{T_{\lambda}^{D}, T_{\lambda}^{A A}\right\}$, then $\lim _{n \rightarrow \infty} T_{i=1}^{\infty} x_{n+i}=1 \Leftrightarrow \sum_{n=1}^{\infty}\left(1-x_{n}\right)^{\lambda}<\infty$.

Note [4, Remark 13] that if $T$ is a $t$-norm for which there exists a sequence $\left(x_{n}\right) \subset[0,1]$ such that $\lim _{n \rightarrow \infty} x_{n}=1$ and $\lim _{n \rightarrow \infty} T_{i=1}^{\infty} x_{n+i}=1$, then $\sup _{t<1} T(t, t)=1$.

\subsection{Menger spaces and generalized Menger spaces. Probabilistic contractions of Sehgal} type. Let $\Delta_{+}$be the class of distance distribution functions [9], that is, the class of all functions $F:[0, \infty) \rightarrow[0,1]$ with the properties
(a) $F(0)=0$;
(b) $F$ is nondecreasing;
(c) $F$ is left continuous on $(0, \infty)$.

$D_{+}$is the subset of $\Delta_{+}$containing the functions $F$ which also satisfy the condition $\lim _{x \rightarrow \infty} F(x)=1$.

A special element of $D_{+}$is the function $\varepsilon_{0}$, defined by

$$
\varepsilon_{0}(t)= \begin{cases}0, & \text { if } t=0 \\ 1, & \text { if } t>0\end{cases}
$$

A sequence $\left(F_{n}\right)$ in $\Delta_{+}$is said to be weakly convergent to $F \in \Delta_{+}$(shortly $F_{n} \stackrel{w}{\longrightarrow} F$ ) if $\lim _{n \rightarrow \infty} F_{n}(x)=F(x)$ for every continuity point $x$ of $F$.

If $X$ is a nonempty set, a mapping $F: X \times X \rightarrow \Delta_{+}$is called a probabilistic distance on $X$ and $F(x, y)$ is denoted by $F_{x y}$.

The triple $(X, F, T)$, where $X$ is a nonempty set, $F$ is a probabilistic distance on $X$, and $T$ is a $t$-norm, is called a generalized Menger space (or a Menger space in the sense of 
Schweizer and Sklar) if the following conditions hold:

$$
\begin{gathered}
F_{x y}=\varepsilon_{0} \Longleftrightarrow x=y, \\
F_{x y}=F_{y x}, \quad \forall x, y \in X, \\
F_{x y}(t+s) \geq T\left(F_{x z}(t), F_{z y}(s)\right), \quad \forall x, y, z \in X, \forall t, s>0 .
\end{gathered}
$$

A Menger space is a generalized Menger space with the property Range $(F) \subset D_{+}$. If $(X, F, T)$ is a generalized Menger space with $\sup _{t<1} T(t, t)=1$, then the family

$$
\left\{U_{\varepsilon, \lambda}\right\}_{\varepsilon>0, \lambda \in(0,1)}, \quad U_{\mathcal{\varepsilon}, \lambda}=\left\{(x, y) \in X \times X: F_{x y}(\varepsilon)>1-\lambda\right\}
$$

is a base for a metrizable uniformity on $X$, named the F-uniformity and denoted by $U_{F}$.

$U_{F}$ naturally determines a topology on $X$, called the F-topology:

$$
O \in \mathscr{T}_{F} \Longleftrightarrow \forall x \in O \exists \varepsilon>0, \exists \lambda \in(0,1) \text { such that } U_{\varepsilon, \lambda}(x) \subset O \text {. }
$$

$U_{F}$ is also generated by the family $\left\{V_{\delta}\right\}_{\delta>0}$ where $V_{\delta}:=U_{\delta, \delta}$. In what follows the topological notions refer to the $F$-topology. Thus, a sequence $\left(x_{n}\right)_{n \in N}$ is $F$-convergent to $x \in X$ if for all $\varepsilon>0, \lambda \in(0,1)$ there exists $k \in N$ such that $F_{x x_{n}}(\varepsilon)>1-\lambda$ for all $n \geq k$.

Definition 1.4. A sequence $\left(x_{n}\right)_{n \in N}$ in $X$ is called F-Cauchy if for each $\varepsilon>0, \lambda \in(0,1)$ there exists $k \in N$ such that $F_{x_{r} x_{s}}(\varepsilon)>1-\lambda$ for all $s \geq r \geq k$.

Probabilistic contractions were first defined and studied by V. M. Sehgal in his doctoral dissertation at Wayne State University.

Definition 1.5 [10]. Let $S$ be a nonempty set and let $F$ be a probabilistic distance on $S$. A mapping $f: S \rightarrow S$ is called a probabilistic contraction (or B-contraction) if there exists $k \in(0,1)$ such that

$$
F_{f(p) f(q)}(k t) \geq F_{p q}(t), \quad \forall p, q \in S, \forall t>0 .
$$

In [10] it is showed that any contraction map on a complete Menger space in which the triangle inequality is formulated under the strongest triangular norm $T_{M}$ has a unique fixed point. In [11] Sherwood showed that one can construct a complete Menger space under $T_{L}$ and a fixed-point-free contraction map on that space. Hadžić [2] introduced the class $\mathcal{H}$ which have the property that Sehgal's result can be extended to any continuous triangular norm in that class. Completing the result of Hadžic, Radu solved the problem of the existence of fixed points for probabilistic contractions in complete Menger spaces $(S, F, T)$ with $T$ continuous. Namely, the following theorem holds.

Theorem 1.6 [7]. Every B-contraction in a complete Menger space (S,F,T) with T continuous has a (unique) fixed point if and only if $T$ is of Hadžić-type.

However, under some additional growth conditions on the probabilistic metric $F$ one may replace the $t$-norm of $H$-type in the above theorem, as in Tardiff's paper [13]. Corollary 2.6 in our paper gives another result in this respect. 


\section{Main results}

The main result of this paper is Theorem 2.4 concerning contractive mappings satisfying an implicit relation similar to that in $[6,12]$. This theorem generalizes the mentioned result of Hadžić (see Corollary 2.7). Note that we work in generalized Menger spaces.

We begin with an auxiliary result, which is formulated as follows.

Lemma 2.1. Let $(X, F, T)$ be a generalized Menger space and let $\left(x_{n}\right)_{n \in N}$ be a sequence in $X$ such that, for some $k \in(0,1)$,

$$
F_{x_{n} x_{n+1}}(k t) \geq F_{x_{n-1} x_{n}}(t), \quad \forall n \geq 1, \forall t>0 .
$$

If there exists $\gamma>1$ such that

$$
\lim _{n \rightarrow \infty} T_{i=n}^{\infty} F_{x_{0} x_{1}}\left(\gamma^{i}\right)=1,
$$

then $\left(x_{n}\right)_{n \in N}$ is an F-Cauchy sequence.

Proof. First note [4] that if the condition $\lim _{n \rightarrow \infty} T_{i=n}^{\infty} F_{x_{0} x_{1}}\left(\gamma^{i}\right)=1$ holds for some $\gamma=$ $\gamma_{0}>1$, then it is satisfied for all $\gamma>1$. Indeed, if $\lim _{n \rightarrow \infty} T_{i=n}^{\infty} F_{x_{0} x_{1}}\left(\gamma_{0}^{i}\right)=1$ and $\gamma \geq \gamma_{0}$, then $\lim _{n \rightarrow \infty} T_{i=n}^{\infty} F_{x_{0} x_{1}}\left(\gamma^{i}\right) \geq \lim _{n \rightarrow \infty} T_{i=n}^{\infty} F_{x_{0} x_{1}}\left(\gamma_{0}^{i}\right)=1$ and therefore $\lim _{n \rightarrow \infty} T_{i=n}^{\infty} F_{x_{0} x_{1}}\left(\gamma^{i}\right)=1$, while if $\gamma<\gamma_{0}$, then $\gamma^{s}>\gamma_{0}$, for some $s \in N$, and now $\lim _{n \rightarrow \infty} T_{i=n+s}^{\infty} F_{x_{0} x_{1}}\left(\gamma^{i}\right) \geq$ $\lim _{n \rightarrow \infty} T_{i=n}^{\infty} F_{x_{0} x_{1}}\left(\gamma_{0}^{i}\right)=1$.

We will prove that

$$
\forall \varepsilon>0, \exists n_{0}=n_{0}(\varepsilon): F_{x_{n} x_{n+m}}(\varepsilon)>1-\varepsilon, \quad \forall n \geq n_{0}, \forall m \in N .
$$

Let $\mu \in(k, 1)$ and let $\delta=k / \mu$. From the above remark it follows that

$$
\lim _{n \rightarrow \infty} T_{i=n}^{\infty} F_{x_{0} x_{1}}\left(\frac{1}{\mu^{i}}\right)=1 .
$$

Let $\varepsilon>0$ be given and $y_{i}:=F_{x_{0} x_{1}}\left(1 / \mu^{i}\right)$. From $\lim _{n \rightarrow \infty} T_{i=1}^{\infty} y_{n+i}=1$ it follows that there exists $n_{1} \in N$ such that $T_{i=1}^{m} y_{n+i-1}>1-\varepsilon$, for all $n \geq n_{1}$, for all $m \in N$.

Since the series $\sum_{n=1}^{\infty} \delta^{n}$ is convergent, there exists $n_{2} \in N$ such that $\sum_{n=n_{2}}^{\infty} \delta^{n}<\varepsilon$.

Let $n_{0}=\max \left\{n_{1}, n_{2}\right\}$. Then, for all $n \geq n_{0}$ and $m \in N$, we have

$$
\begin{aligned}
F_{x_{n} x_{n+m}}(\varepsilon) & \geq F_{x_{n} x_{n+m}}\left(\sum_{i=n}^{n+m-1} \delta^{i}\right) \\
& \geq T_{i=0}^{m-1} F_{x_{n+i} x_{n+i+1}}\left(\delta^{n+i}\right) \geq T_{i=0}^{m-1} y_{n+i}>1-\varepsilon,
\end{aligned}
$$

where the last " $\geq$ " inequality follows from $F_{x_{s} x_{s+1}}\left(\delta^{s}\right)=F_{x_{s} x_{s+1}}(k / \mu)^{s} \geq F_{x_{0} x_{1}}\left(1 / \mu^{s}\right)$ for all $s \geq 1$, which immediately can be proved by induction.

In the following we deal with the class $\Phi$ of all continuous functions $\varphi:[0,1]^{4} \rightarrow \mathbb{R}$ with the property:

$$
\varphi(u, v, v, u) \geq 0 \Longrightarrow u \geq v .
$$

Next we give some examples of functions in $\Phi$. 
Example 2.2. If $a, b, c, d \in \mathbb{R}$ and $a+b+c+d=0$, then $\varphi\left(t_{1}, t_{2}, t_{3}, t_{4}\right):=a t_{1}+b t_{2}+c t_{3}+$ $d t_{4} \in \Phi$ if and only if $a+d>0$.

Indeed, $a+d \leq 0 \Rightarrow b+c \geq 0$. Choosing $u=0, v=1$ we have $u<v$ and $\varphi(u, v, v, u)=$ $(a+d) u+(b+c) v=b+c \geq 0$.

Conversely, if $a+d>0$ and $\varphi(u, v, v, u) \geq 0$, then $(a+d) u \geq-(b+c) v$, that is $(a+$ d) $u \geq(a+d) v$, which implies that $u \geq v$.

Thus, the functions $\varphi_{1}, \varphi_{2}$,

$$
\begin{aligned}
& \varphi_{1}\left(t_{1}, t_{2}, t_{3}, t_{4}\right)=t_{1}-t_{2}, \\
& \varphi_{2}\left(t_{1}, t_{2}, t_{3}, t_{4}\right)=t_{1}-t_{3},
\end{aligned}
$$

are in $\Phi$.

Also, the function $\varphi$ defined by $\varphi\left(t_{1}, t_{2}, t_{3}, t_{4}\right)=t_{1}^{2}-t_{2} t_{3}$ and, more generally, $\varphi\left(t_{1}, t_{2}\right.$, $\left.t_{3}, t_{4}\right)=t_{1}^{2}-\left(a t_{2}^{2}+b t_{3}^{2}\right)-t_{2} t_{3}$ with $a+b=0$ are in $\Phi$.

In the proof of Theorem 2.4 we need the following lemma, which is the analog of uniform continuity of a metric (note that $([0,1], T)$ is rather a semigroup than a group).

Lemma 2.3. Let $(S, F, T)$ be a generalized Menger space with $T$ continuous in $(a, 1)$ for all $a \in(0,1)$, that is,

$$
\lim _{n \rightarrow \infty} a_{n}=a, \quad \lim _{n \rightarrow \infty} b_{n}=1 \Longrightarrow \lim _{n \rightarrow \infty} T\left(a_{n}, b_{n}\right)=a .
$$

If $p, q \in S$ and $\left(p_{n}\right)$ is a sequence in $S$ such that $p_{n} \rightarrow p$, then $F_{p_{n} q} \stackrel{w}{\longrightarrow} F_{p q}$.

Proof. Let $p, q \in S, p_{n} \rightarrow p$ and $t$ be a continuity point of $F_{p q}$. By (1.7) it follows that for all $0<\varepsilon<t$,

$$
\begin{aligned}
& F_{p_{n} q}(t) \geq T\left(F_{p_{n} p}(\varepsilon), F_{p q}(t-\varepsilon)\right), \\
& F_{p q}(t+\varepsilon) \geq T\left(F_{p_{n} p}(\varepsilon), F_{p_{n} q}(t)\right) .
\end{aligned}
$$

Therefore, $\lim _{n} \inf F_{p_{n} q}(t) \geq F_{p q}(t-\varepsilon)$ and $F_{p q}(t+\varepsilon) \geq \lim _{n} \sup F_{p_{n} q}(t)$. Letting $\varepsilon \rightarrow 0$ we obtain $\lim _{n} \sup F_{p_{n} q}(t) \leq F_{p q}(t) \leq \lim _{n} \inf F_{p_{n} q}(t)$, and thus $\lim _{n \rightarrow \infty} F_{p_{n} q}(t)=F_{p q}(t)$.

Theorem 2.4. Let $(X, F, T)$ be an F-complete generalized Menger space under a $t$-norm $T$ which is continuous in $(a, 1)$ for all $a \in(0,1), k \in(0,1)$, and $\varphi \in \Phi$. If $f: X \rightarrow X$ is a mapping such that

$$
\left(\varphi_{f}\right): \varphi\left(F_{f(x) f(y)}(k t), F_{x y}(t), F_{x f(x)}(t), F_{y f(y)}(k t)\right) \geq 0, \quad \forall x, y \in X, \forall t>0
$$

and there exist $x_{0} \in X$ and $\gamma>1$ for which $\lim _{n \rightarrow \infty} T_{i=n}^{\infty} F_{x_{0}} f\left(x_{0}\right)\left(\gamma^{i}\right)=1$, then $f$ has a fixed point.

Proof. Let $x_{0} \in X$ be such that $\lim _{n \rightarrow \infty} T_{i=n}^{\infty} F_{x_{0}} f\left(x_{0}\right)\left(\gamma^{i}\right)=1$ and, for all $n \geq 1, x_{n}=f\left(x_{n-1}\right)$. Note that $\left(\varphi_{f}\right)$ implies that

$$
F_{f(x) f^{2}(x)}(k t) \geq F_{x f(x)}(t), \quad \forall x \in X, \forall t>0 .
$$


On taking in this relation $x=x_{n}$ we obtain

$$
\varphi\left(F_{x_{n+1} x_{n+2}}(k t), F_{x_{n} x_{n+1}}(t), F_{x_{n} x_{n+1}}(t), F_{x_{n+1} x_{n+2}}(k t)\right) \geq 0, \quad \forall n \in N, \forall t>0 .
$$

It follows that $F_{x_{n+1} x_{n+2}}(k t) \geq F_{x_{n} x_{n+1}}(t)$, for all $n \in N$, for all $t>0$ and therefore, by Lemma 2.1, $\left(x_{n}\right)$ is a Cauchy sequence.

By the $F$-completeness of $X$ it follows that there exists $u \in X$ such that $\lim _{n \rightarrow \infty} F_{u x_{n}}(t)=$ 1 , for all $t>0$.

Notice that from $F_{x_{n+1} x_{n+2}}(k t) \geq F_{x_{n} x_{n+1}}(t)$, for all $n \in N$, for all $t>0$ it follows that $\lim _{n \rightarrow \infty} F_{x_{n} x_{n+1}}(t)=1$, for all $t>0$, for $\lim _{n \rightarrow \infty} T_{i=n}^{\infty} F_{x_{0}} f\left(x_{0}\right)\left(\gamma^{i}\right)=1$ implies that $\lim _{n \rightarrow \infty} F_{x_{0} f\left(x_{0}\right)}\left(\gamma^{n}\right)=1$ (therefore $F_{x_{0} f\left(x_{0}\right)} \in D_{+}$) and $F_{x_{n} x_{n+1}}(t) \geq F_{x_{0} x_{1}}\left(t / k^{n}\right)$, for all $n \in N$, for all $t>0$.

Next, on taking $x=x_{n}, y=u$ in $\left(\varphi_{f}\right)$ one obtains

$$
\varphi\left(F_{x_{n+1} f(u)}(k t), F_{x_{n} u}(t), F_{x_{n} x_{n+1}}(t), F_{u f(u)}(k t)\right) \geq 0, \quad \forall n \in N, \forall t>0 .
$$

If $k t$ is a continuity point of $F_{u f(u)}$, then, on taking $n \rightarrow \infty$ in the above inequality and using Lemma 2.3, we get

$$
\varphi\left(F_{u f(u)}(k t), 1,1, F_{u f(u)}(k t)\right) \geq 0
$$

Thus $F_{u f(u)}(k t)=1$. Since $F_{u f(u)}$ is increasing, the set of its discontinuity points is at most countable. Hence $F_{u f(u)}(k t)=1$ for all $t>0$, from which (using $\left.(1.5)\right)$ we obtain $u=f(u)$. This completes the proof.

Corollary 2.5 [5, Theorem 2.1]. Let $(X, F, T)$ be an F-complete generalized Menger space under a continuous $t$-norm $T \in \mathcal{H}, k \in(0,1)$, and $\varphi \in \Phi$. If $f: X \rightarrow X$ is a mapping such that

$$
\varphi\left(F_{f(x) f(y)}(k t), F_{x y}(t), F_{x f(x)}(t), F_{y f(y)}(k t)\right) \geq 0, \quad \forall x, y \in X, \forall t>0
$$

and there exists $x_{0} \in X$ for which $F_{x_{0} f\left(x_{0}\right)} \in D_{+}$, then $f$ has a fixed point.

Proof. Choose a $\mu>1$. Since $\lim _{n \rightarrow \infty} \mu^{n}=\infty$ and $F_{x_{0} x_{1}} \in D_{+}$, it follows that $\lim _{n \rightarrow \infty} F_{x_{0} f\left(x_{0}\right)}\left(\mu^{n}\right)=1$. Therefore, by Proposition 1.3(iii),

$$
\lim _{n \rightarrow \infty} T_{i=n}^{\infty} F_{x_{0} f\left(x_{0}\right)}\left(\mu^{i}\right)=1
$$

Now apply Theorem 2.4.

Corollary 2.6. Let $\left(X, F, T_{L}\right)$ be an F-complete generalized Menger space and $\varphi \in \Phi$. If $f: X \rightarrow X$ is a mapping such that

$$
\varphi\left(F_{f(x) f(y)}(k t), F_{x y}(t), F_{x f(x)}(t), F_{y f(y)}(k t)\right) \geq 0, \quad \forall x, y \in X, \forall t>0,
$$

and $\sum_{n=1}^{\infty}\left(1-F_{x_{0} f\left(x_{0}\right)}\left(\gamma^{n}\right)\right)<\infty$ for some $x_{0} \in X$ and $\gamma>1$, then $f$ has a fixed point.

For the proof see Proposition 1.3. 
Corollary 2.7. Let $(X, F, T)$ be an F-complete generalized Menger space under $T \in$ $\left\{T_{\lambda}^{D}, T_{\lambda}^{A A}\right\}, k \in(0,1)$, and $\varphi \in \Phi$. If $f: X \rightarrow X$ is a mapping such that

$$
\varphi\left(F_{f(x) f(y)}(k t), F_{x y}(t), F_{x f(x)}(t), F_{y f(y)}(k t)\right) \geq 0, \quad \forall x, y \in X, \forall t>0
$$

and $\sum_{n=1}^{\infty}\left(1-F_{x_{0} f\left(x_{0}\right)}\left(\gamma^{n}\right)\right)^{\lambda}<\infty$ for some $x_{0} \in X$ and $\gamma>1$, then $f$ has a fixed point.

CoRollary 2.8. Let $(X, F, T)$ be an F-complete generalized Menger space under a continuous $t$-norm $T \in \mathcal{H}$ and $k \in(0,1)$. If $f: X \rightarrow X$ is a mapping satisfying one of the following conditions:

$$
\begin{gathered}
F_{f(x) f(y)}(k t) \geq F_{x y}(t), \quad \forall x, y \in X, \forall t>0, \\
F_{f(x) f(y)}^{2}(k t) \geq F_{x y}(t) F_{x f(x)}(t), \quad \forall x, y \in X, \forall t>0, \\
F_{f(x) f(y)}(k t) \geq 2 F_{x y}(t)-F_{x f(x)}(t), \quad \forall x, y \in X, \forall t>0
\end{gathered}
$$

and there exists $x_{0} \in X$ for which $F_{x_{0} f\left(x_{0}\right)} \in D_{+}$, then $f$ has a fixed point.

As a final result for this section, we consider an example to see the generality of Theorem 2.4 .

Example 2.9. Let $X$ be a set containing at least two elements and the mapping $F$ from $X \times X$ to $\Delta_{+}$, defined by

$$
F_{x y}(t)=\left\{\begin{array}{ll}
0, & \text { if } t \leq 1 \\
\frac{1}{2}, & \text { if } t>1
\end{array} \quad \text { for } x, y \in X, x \neq y, \quad F_{x x}=\varepsilon_{0}, \quad \forall x \in X\right.
$$

It is easy to show (see [14]) that $\left(X, F, T_{M}\right)$ is a complete Menger space.

We are going to prove that the mapping $f: X \rightarrow X, f(x)=x$ satisfies the contractivity condition (2.21) from the above corollary with $b=2, c=-1$, however it is not a $B$-contraction (here we took advantage of working in $\Delta_{+}$rather than in $D_{+}$).

First, we show that

$$
F_{x y}(k t)+1 \geq 2 F_{x y}(t), \quad \forall x, y \in X, \forall t>0 .
$$

Indeed, the above inequality holds with equality if $x=y$, while if $x \neq y$ then the righthand member is at most 1 .

Next, for every $t \in(1,1 / k], F_{x y}(k t)=0$, while $F_{x y}(t)=1 / 2$, which means that $f$ is not a Sehgal contraction.

\section{References}

[1] G. Constantin and I. Istrățescu, Elements of Probabilistic Analysis with Applications, Mathematics and Its Applications (East European Series), vol. 36, Editura Academiei, Bucharest; Kluwer Academic Publishers, Dordrecht, 1989.

[2] O. Hadžić, A generalization of the contraction principle in probabilistic metric spaces, Univ. $\mathrm{u}$ Novom Sadu Zb. Rad. Prirod.-Mat. Fak. 10 (1980), 13-21 (1981).

[3] O. Hadžić and E. Pap, Fixed Point Theory in Probabilistic Metric Spaces, Mathematics and Its Applications, vol. 536, Kluwer Academic Publishers, Dordrecht, 2001. 
[4] New classes of probabilistic contractions and applications to random operators, Fixed Point Theory and Applications (Chinju/Masan, 2001), vol. 4, Nova Science Publishers, New York, 2003, pp. 97-119.

[5] D. Miheț, A generalization of a contraction principle in probabilistic metric spaces, The 9th International Conference on Applied Mathematics and Computer Science, Cluj-Napoca, 2004.

[6] V. Popa, Fixed points for non-surjective expansion mappings satisfying an implicit relation, Bul. Ştiinţ. Univ. Baia Mare Ser. B Fasc. Mat.-Inform. 18 (2002), no. 1, 105-108.

[7] V. Radu, Some fixed point theorems in probabilistic metric spaces, Stability Problems for Stochastic Models (Varna, 1985), Lecture Notes in Math., vol. 1233, Springer-Verlag, Berlin, 1987, pp. 125-133.

[8] Lectures on Probabilistic Analysis, Surveys, Lecture Notes and Monographs. Series on Probability, Statistics and Applied Mathematics, vol. 2, Universitatea din Timişoara, Timişoara, 1994.

[9] B. Schweizer and A. Sklar, Probabilistic Metric Spaces, North-Holland Series in Probability and Applied Mathematics, North-Holland Publishing, New York, 1983.

[10] V. M. Sehgal and A. T. Bharucha-Reid, Fixed points of contraction mappings on probabilistic metric spaces, Math. Systems Theory 6 (1972), 97-102.

[11] H. Sherwood, Complete probabilistic metric spaces, Z. Wahrscheinlichkeitstheorie und Verw. Gebiete 20 (1971/72), 117-128.

[12] B. Singh and S. Jain, A quantitative generalization of Banach contractions, in preparation.

[13] R. M. Tardiff, Contraction maps on probabilistic metric spaces, J. Math. Anal. Appl. 165 (1992), no. 2, 517-523.

[14] E. Thorp, Best possible triangle inequalities for statistical metric spaces, Proc. Amer. Math. Soc. 11 (1960), 734-740.

Dorel Miheţ: Faculty of Mathematics and Computer Science, West University of Timisoara, Bd. V. Parvan 4, 300223 Timisoara, Romania

E-mail address: mihet@math.uvt.ro 


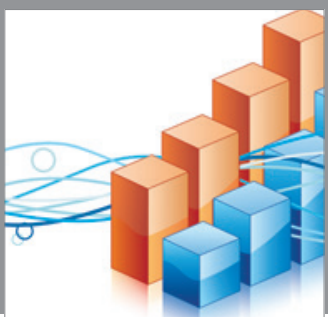

Advances in

Operations Research

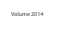

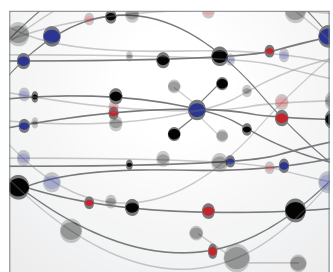

\section{The Scientific} World Journal
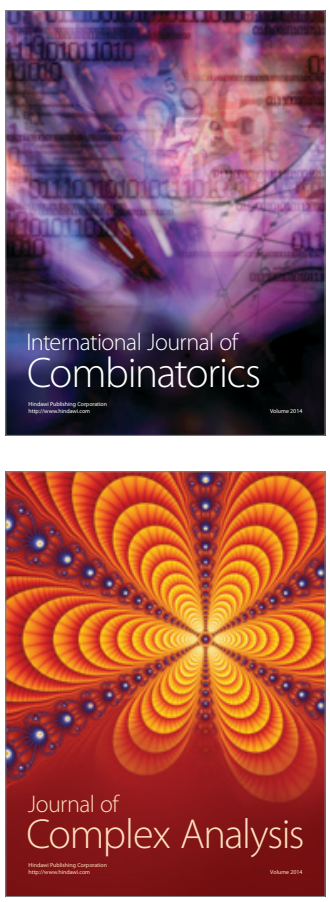

International Journal of

Mathematics and

Mathematical

Sciences
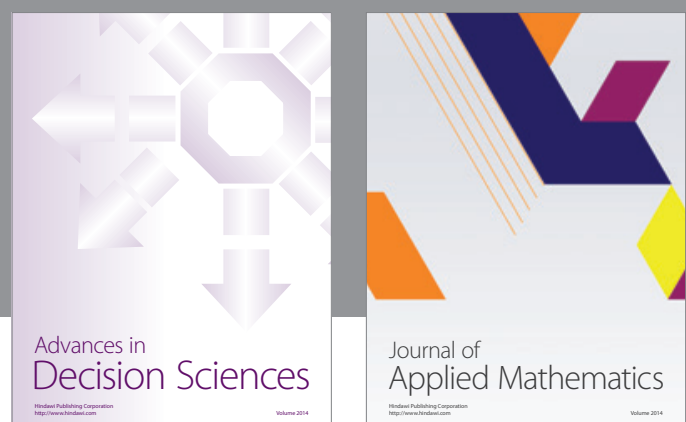

Journal of

Applied Mathematics
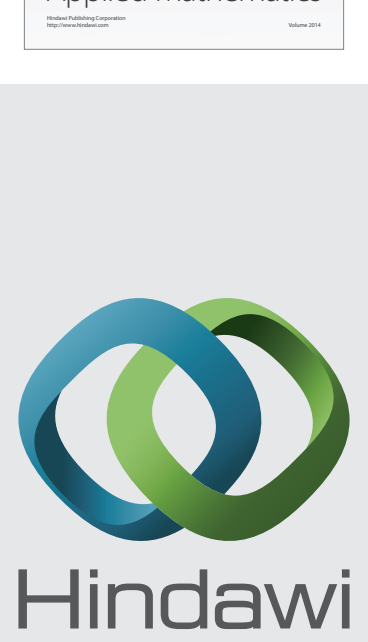

Submit your manuscripts at http://www.hindawi.com
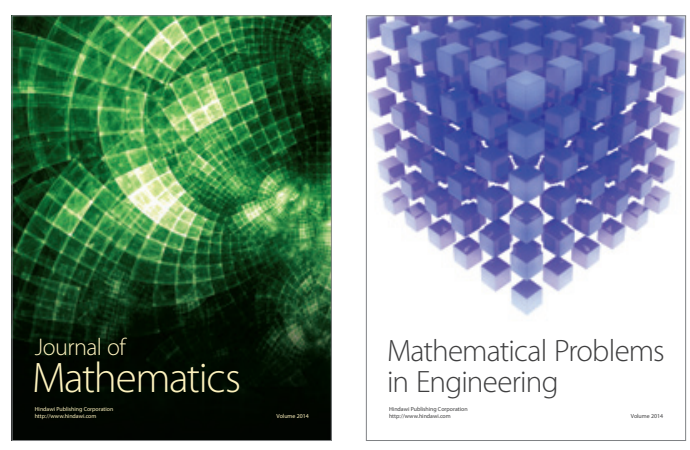

Mathematical Problems in Engineering
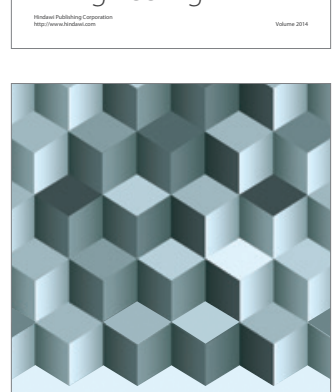

Journal of

Function Spaces
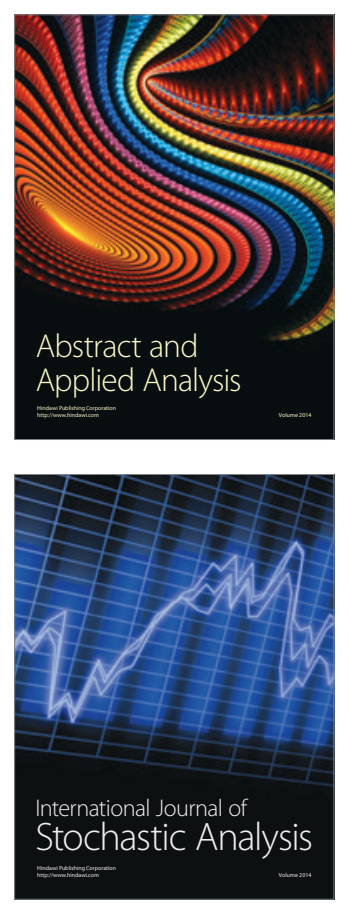

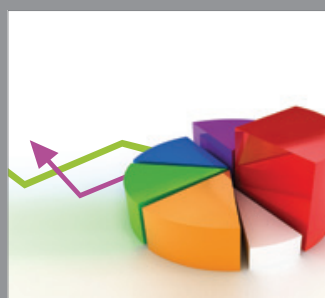

ournal of

Probability and Statistics

Promensencen
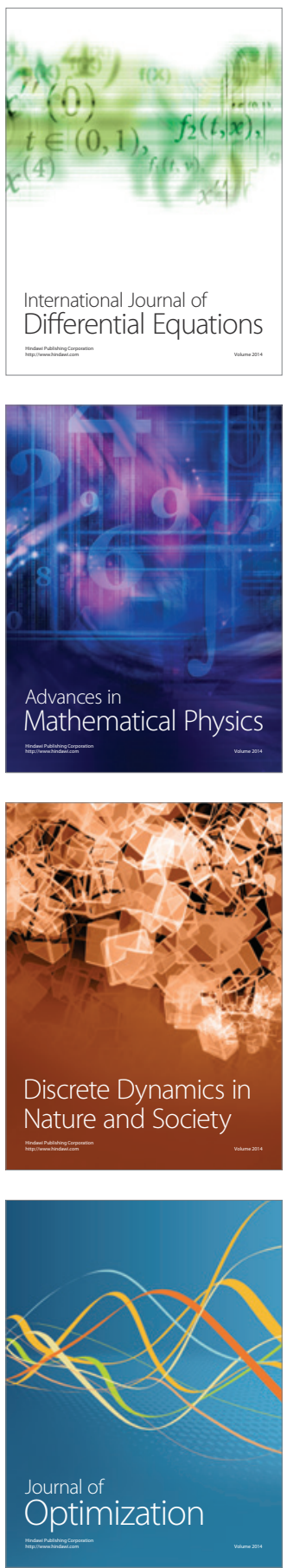